\title{
Innovaciones en la formación del profesorado de matemáticas para contextos multiculturales ${ }^{1}$
}

\author{
Innovations in the mathematics teachers training for multicultural contexts
}

William Oswaldo Flores López²

\section{Resumen}

En este artículo ha descrito las experiencias de innovación en la formación del profesorado de matemáticas en contextos multiculturales. Fue un estudio cualitativo con una perspectiva de teoría fundamentada donde se realizó un análisis hermenéutico de políticas, leyes, normativas, reglamentos, programas curriculares, libros, artículos científicos, artículos de prensa y proyectos de investigación, desarrollo e innovación que han contribuido a la formación del profesorado de matemática. Los resultados han certificado que la formación del profesorado es una estrategia de autodesarrollo regional para contrarrestar las condiciones de pobreza y marginación de los pueblos indígenas, afrodescendientes y mestizo de Nicaragua. Además, que las tecnologías han aportado para que el estudiantado interactúe e intercambie experiencias en un ambiente de aprendizaje innovador y colaborativo. Finalmente, enseñar y aprender matemáticas en contextos multiculturales ha significado la revitalización e implementación de prácticas matemáticas de la vida cotidiana en una comunidad de aprendizaje desde una perspectiva intercultural de género y el reconocimiento de la diversidad de las poblaciones, tomando como referencia la resolución de problemas.

Palabras clave: Brechas-educativas; innovación; formación del profesorado; matemáticas; tecnología; enseñar-aprender.

\section{Abstract}

In this article, it is described the experiences of innovation in the training of mathematics teachers in multicultural contexts. It was a qualitative study with a perspective of grounded theory where a hermeneutical analysis of policies, laws, regulations, curricular programs, books, scientific articles, press articles and research, development and innovation projects that have contributed to the mathematics teachers training was carried out. The results have certified that the teachers training is a regional self-development strategy to respond to the conditions of poverty and marginalization of the indigenous, afro-descendant and mestizo peoples of Nicaragua. In addition, the technologies have contributed for the students to interact and exchange experiences in an innovative and collaborative learning environment. Finally, teaching and learning mathematics in multicultural contexts has meant the revitalization and implementation of mathematical practices of daily life in a learning community from an intercultural perspective of gender and the recognition of the diversity of populations, taking as a reference the resolution of problems.

Keywords: Educational gaps; innovation; teachers training; mathematics; technology; teaching - learning.

\footnotetext{
$\overline{1}$ XIV Congreso Latinoamericano y del Caribe de Extensión Universitaria y Segundo Congreso Centroamericano de Compromiso Social para la vinculación social de la universidad con la sociedad.

2 Doctor en Educación. Profesor Investigador de la Universidad de las Regiones Autónomas de la Costa Caribe Nicaragüense. Correo: william.flores@uraccan.edu.ni ORCID: https://orcid.org/0000-0002-1016-1620
}

Recibido: 17/01/2018 - Aprobado: 05/04/2018

Flores, W., 0. (2018). Innovaciones en la formación del profesorado de matemáticas para contextos multiculturales. Revista Universitaria del Caribe, $20(1)$, 7-22. Dol:http://dx.doi.org/10.5377/ruc.v1i1.6761. 


\section{Introducción}

En esta comunicación se describe las experiencias de innovación en la formación del profesorado de matemáticas para poblaciones en contextos multiculturales. Es decir, la investigación detalla la formación integral desde una perspectiva de aprendizaje intencionado, tendiente al fortalecimiento de una personalidad responsable, ética, crítica, participativa, creativa, solidaria, y con capacidad de reconocer e interactuar con su entorno para que construya su identidad cultural. Busca promover el crecimiento humano a través de un proceso que supone una visión multidimensional de la persona, y tiende a desarrollar aspectos como la inteligencia emocional, intelectual, social, material, ética, actitudes y valores.

Entonces, la educación en contextos multiculturales enfrenta el reto de contribuir en la desaparición de toda forma de exclusión, discriminación y marginación por disparidad y desigualdad, apoyando en desventaja o marginación, incluyendo las necesidades de las personas con discapacidad (ACACIA, 2015). Es por ello, que la formación del profesorado para contextos multiculturales permite garantizar una educación inclusiva, equitativa y de calidad a través de oportunidades de aprendizaje durante toda la vida para todos y todas (CEPAL, 2016), en ambientes de aprendizaje que tratan de disminuir las disparidades de género en la educación y asegurar el acceso igualitario a todos los niveles de enseñanza y la formación profesional para los pueblos indígenas, afrodescendientes y mestizos de la Costa Caribe de Nicaragua. En este sentido, esta investigación analiza la formación en contextos multiculturales; formación en máster didáctica de las matemáticas; incorporación de tecnología; enseñar y aprender matemáticas y referentes curriculares, con la finalidad de generar teoría fundamentada a partir del análisis hermenéutico de los documentos.

\section{Revisión de literatura}

Las brechas educativas de Nicaragua se pueden observar en promedios de años de escolaridad, el cual figura con un 5,8\%, el segundo más bajo en la subregión (Eduquemos, 2011). Los promedios en la RACCS y RACCN son aún más bajos en 2,9 y 2,3 años, respectivamente. En la RACCS, el $45 \%$ de los niños en edad escolar y el $40 \%$ de las niñas no están en la escuela, y el $25,6 \%$ de las niñas y el $25,2 \%$ de los niños son analfabetos. Además, la tasa de abandono del nivel primario nacional es del 9,5 por ciento, mientras que es del 17 por ciento en la RACCS y cerca del 15 por ciento en la RACCN (Flórez, 2012). En relación a la juventud de la Costa Caribe nicaragüense hay mayor paridad de género en el absentismo escolar que en el nacional (USAID, 2012). En la RACCS y RACCN sólo 43 y 42 por ciento de todos los jóvenes de 15 a 19 años de edad que terminó la escuela primaria en 2010 en comparación con el promedio nacional de $65 \%$ (Eduquemos, 2014). En ambas Regiones de la Costa Caribe, sólo dos de cada diez jóvenes con edad adecuada asisten al primer ciclo de secundaria y para el segundo ciclo sólo uno de cada diez jóvenes asiste a la escuela "las tasas más bajas del país" (USAID, 2013).

En relación con la Educación Superior, en el año 2015, las universidades del Consejo Nacional de Universidades (CNU) tuvieron una matrícula total de 111,572 estudiantes, Técnico Superior 989; Profesorado de Educación Media 830; y Licenciaturas/Ingenierías 109.753 estudiantes. De este total de estudiantes, el 9,75\% son hombres y mujeres procedentes de comunidades de la Costa Caribe nicaragüense (CNU, 2016). Sin embargo, el 30\% de esta población estudiantil tiende a abandonar sus estudios universitarios por indicadores relacionados con aspectos como: académicos, económicos, motivacionales, normativas, ambiente familiar, orientación profesional, absentismo por género (Femenino), étnico, lingüísticos y procesos de acompañamientos al estudiantado en su formación (Flores et al., 2016).

En la Educación Superior de la Costa Caribe nicaragüense también se observa una brecha de género, ya que hombres se desempeñan mejor en materias difíciles como matemáticas y ciencias, y las mujeres en temáticas con sesgos culturales y educativos en relacionan con carreras y asignaturas de Ciencias, Tecnología, Ingeniería y Matemáticas (USAID, 2012). Igualmente, en los estudios de seguimientos de graduados se observa que 1132 graduados de URACCAN el 62\% son mujeres; sin embargo, sigue prevaleciendo la brecha de género, ya que los hombres se han graduado en ingenierías y matemáticas, y las mujeres en carreras relacionadas con las ciencias sociales, humanidades e idiomas (URACCAN, 2010). 


\section{Materiales y métodos}

Esta investigación es de carácter cualitativa porque es una actividad sistemática orientada a la comprensión en profundidad de fenómenos educativos y sociales a la transformación de prácticas y escenarios socioeducativos a la toma de decisiones y también hacia el descubrimiento y desarrollo de un cuerpo organizado de conocimiento (Sandín, 2003, p. 123). Sabiendo que, el diseño en la investigación cualitativa, es el abordaje general que se utilizará en el proceso de investigación (Hernández, Fernández, \& Baptista, 2010). Es por ello, que este se aborda desde la teoría fundamentada, porque es comprensiva e interpretativa en cuanto su principal finalidad es construir teoría para explicar la realidad sobre la base de la recogida y el análisis sistemático de datos (Bisquerra, 2009).

En este sentido, esta investigación trata de describir las innovaciones en la formación del profesorado de matemáticas en contextos multiculturales, a partir de un análisis hermenéutico de las variables: formación de profesores de matemáticas y máster en didácticas de las matemáticas; enseñar y aprender matemáticas en contextos multiculturales; incorporación de tecnología; y referentes curriculares para la formación de profesores.

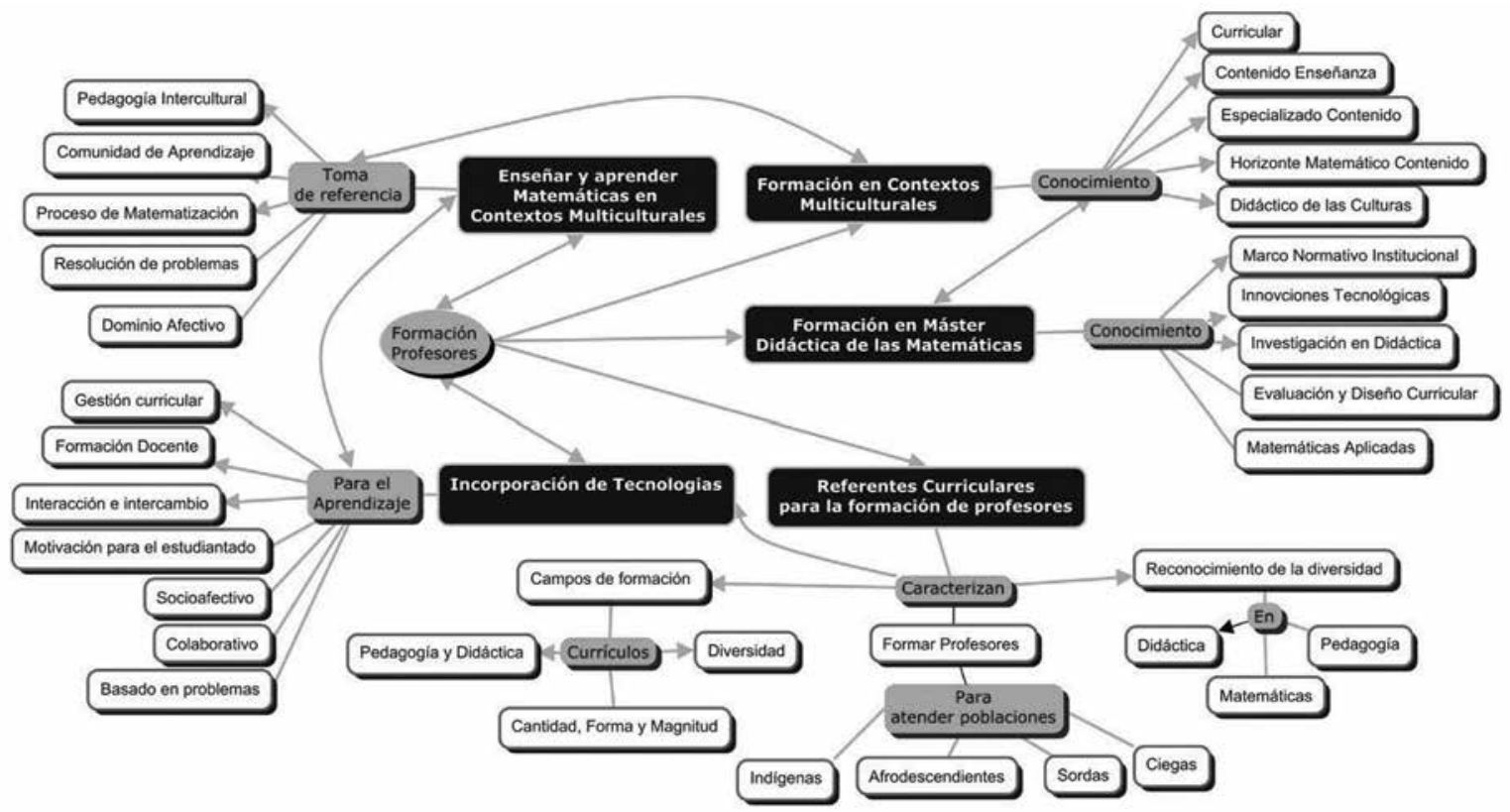

Figura 1: Variables en la formación del profesorado de matemáticas en contextos multiculturales.

Es por ello, que se realiza una revisión de documentos, donde nos centramos en los argumentos, las razones, los propósitos, y los significados que los documentos presentan en la formación de profesores. Por lo tanto, se construyó una guía de revisión documental que permitió describir las experiencias reflejadas en políticas, leyes, normativas, reglamentos, programas curriculares, libros, artículos científicos, artículos de prensa y proyectos de investigación, desarrollo e innovación sobre la formación del profesorado de matemáticas en contextos multiculturales.

\section{Resultados}

\section{Formación del profesorado de matemáticas para contextos multiculturales}

Aprender a enseñar matemáticas es un proceso paulatino de incorporación en la comunidad de práctica, diferenciando dos tipos de prácticas íntimamente relacionadas: la relativa a la matemática y la relativa a la didáctica. Dichas prácticas dependen del contexto cultural y social en el cual se participa, puesto 
que las instituciones culturales proveen a las comunidades de prácticas los instrumentos físicos y conceptuales con los cuales realizar las actividades propias de la práctica de enseñar, además de las formas de realizarlas; es decir, las maneras de explorar, de decir, de comunicar, de ser. Así el estudiantado para profesor desarrolla una experiencia con un cierto tipo de conocimiento característicos de su profesión.

El conocimiento profesional del profesor de matemáticas. En qué consiste este conocimiento y la definición de sus componentes, sigue siendo una de las preocupaciones de los investigadores en matemáticas. También, cobra interés lo propuesto por Shulman (1986), denomino conocimiento de la materia a enseñar otros han llamado matemáticas para enseñar o conocimiento matemático para enseñar. Ball, Thames y Phelps (2008) caracterizan el conocimiento del profesor de matemáticas como un conocimiento de carácter práctico profesional; conjugan el conocimiento de la materia (matemáticas) y el contenido pedagógico (Shulman, 1986), y lo denominan conocimiento matemático para enseñar, como saber necesario para realizar la práctica de enseñar matemáticas. Desde una formación matemática para contextos multiculturales es necesario contemplar, además, el conocimiento del entorno cultural del aula.

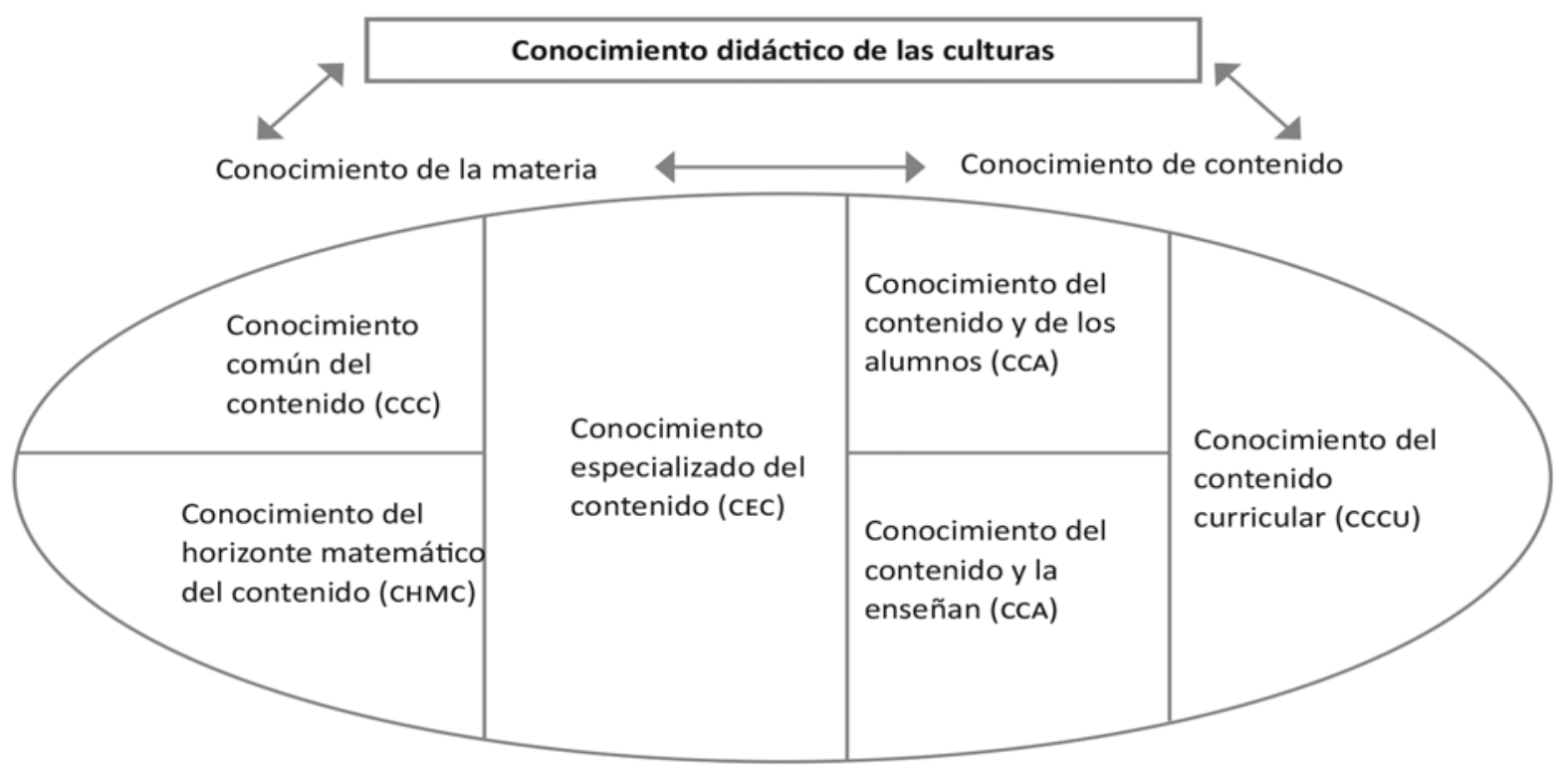

Figura 2: Conocimiento pedagógico-didáctico del profesor (Ball et al., 2008)

- Conocimiento común del contenido. Es el conocimiento matemático y habilidades usadas en contextos diferentes a la enseñanza.

- Conocimiento especializado del contenido. Es el conocimiento matemático y habilidades que son propias/únicas de la enseñanza.

- Conocimiento del horizonte matemático del contenido. Es una conciencia de cómo los temas matemáticos están relacionados a lo largo del currículo de matemáticas.

- Conocimiento del contenido y de la enseñanza. Combina el conocimiento sobre matemáticas y su enseñanza. Muchas de las tareas matemáticas en la enseñanza requieren un conocimiento matemático del diseño y de la instrucción.

- Conocimiento curricular. Representado por la amplia gama de programas diseñados para la enseñanza de temas específicos y de tópicos matemáticos en un nivel dado, la variabilidad de materiales didácticos disponibles en relación con estos programas y el conjunto de características que sirven como indicaciones y contradicciones para el uso de esos materiales didácticos en los currículos particulares o en circunstancias específicas. 
- Conocimiento didáctico de las culturas. Es el conocimiento de potencial didáctico que las culturas confluyen y constituyen el entorno social del aula ofrecen al proceso de enseñanza de las matemáticas. En este marco el uso de las TIC en la enseñanza de las matemáticas es considerado como un aspecto a abordar dentro del conocimiento del contenido y la enseñanza; su incorporación tiene que ver con la reflexión sobre las posibilidades de múltiples representaciones y medios de comunicación y exploración matemáticas.

La experiencia de formación del profesorado para contextos multiculturales en la Universidad Comunitaria Intercultural se caracteriza por la propuesta de las competencias siguientes: currículo diversificado, coherente y con distintos modelos de aprendizaje; trabajar en matemática pensando y comprendiendo; la resolución de problemas como estrategias de enseñanza y aprendizaje; dominio de diversos procedimientos de evaluación; percepción y aceptación del binomio matemática e internet; conocimiento y dominio de la matemática como ciencia en los campos estructurantes (Flores, 2015):

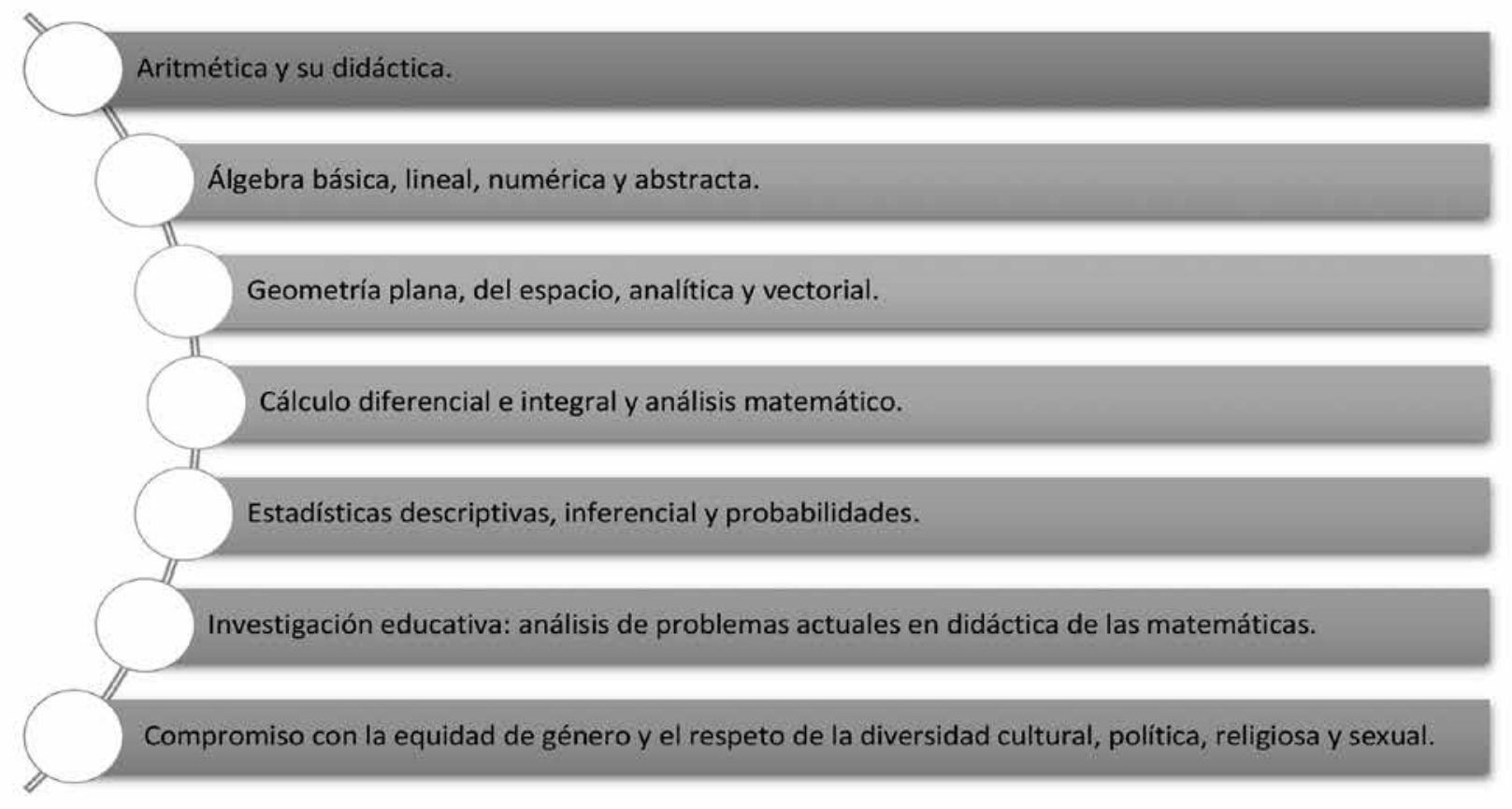

Figura 3: Campos estructurantes en la formación de profesores de matemáticas de la URACCAN.

Algo importante a mencionar es que la diversidad del estudiantado y del profesorado no es ajena a la clase de matemáticas, pues en ella confluyen varios tipos de diversidades: la de los protagonistas del hecho educativo, manifestado en constituciones de subjetividad diversas; la de los escenarios de exploración matemática; la de los sistemas, los instrumentos para la exploración y la reflexión matemática, los sistemas semióticos de representación (las lenguas, los sistemas de numeración, los sistemas simbólicos desarrollados para cada campo de conocimiento matemático); la de los momentos de elaboración de conocimientos matemáticos y los dispositivos didácticos que dinamizan esos momentos. La anterior diversidad individual enriquece en la sociedad ofreciendo un ambiente de enseñanza y aprendizaje matizado, donde los participantes del acto educativo comparten su propósitos, intereses, capacidades y contextos (León, 2014).

- El reconocimiento de la diversidad en matemáticas, como señalan Martín, Dolz y Mauri (1997), permite aprovechar los distintos saberes que se encuentran en el aula, incorporar los contextos socioculturales a los procesos de enseñanza y aprendizaje, expandir los elementos de resolución de problemas y hallar sentido a los aprendizajes que posteriormente se transfieran a la vida cotidiana. El profesorado es un diseñador, un arquitecto que va construyendo y solidificando su interacción 
con los grupos facilitando la adquisición de aspectos disciplinares de las matemáticas respecto a las dinámicas que se van generando. De este modo, el profesorado de matemáticas para contextos multiculturales tiene que cumplir las características siguientes: 1) Creativo y emprendedor; 2) Investigador e innovador; 3) Facilitador y colaborativo; 4) Gestor de conocimientos, saberes y prácticas en comunidad.

- Cuando se habla de profesor creativo y emprendedor, es aquel profesorado que tiene la capacidad de generar nuevas ideas y asociarlas con la ya conocidas, y así fomentar en el estudiantado el pensamiento creativo, es decir, que sea capaz de promover el análisis y la reflexión, lo que conlleva a que el estudiantado genere múltiples ideas sobre el conocimiento que está aprendiendo.

- El profesorado investigador e innovador, es capaz de profundizar en el estudio de las tipologías de aprendizaje de sus estudiantes, es decir, el profesor investigador genera nuevas metodologías de aprendizaje para que el estudiantado adquiera conocimientos. Usa las tecnologías de la información y comunicación para innovar y transformar el aprendizaje, promoviendo la autonomía de sus estudiantes y la riqueza de aprender un nuevo conocimiento a través de la investigación.

- El profesorado facilitador y colaborador, es aquel profesor que facilita la construcción del conocimiento a través de la colaboración e interacción con el estudiantado. El profesor facilita nociones teóricas y prácticas para la interacción con el estudiantado, lo que conlleva al estudiantado a crear y recrear su propio aprendizaje.

- El profesorado gestor de conocimientos, saberes y prácticas en comunidad acompaña y facilita al estudiantado el aprendizaje a partir de las habilidades, conocimientos y actitudes que le cultiva día a día, además se centra en cómo aprende el estudiantado y como la comunidad le contribuye a comprender, analizar, sintetizar, aplicar y evaluar los saberes y prácticas con el mundo real.

Estas características generan un impacto emocional porque inciden en la motivación del estudiantado, también estimula la autoevaluación y el aprendizaje autónomo, el cual juega un papel central en la actividad en el aula. Así mismo, estas características promueven un compromiso con los resultados de los aprendizajes esperados que conlleva a mejorar y reconocer los logros del estudiantado.

\section{Formación del profesorado en el grado de Máster en Didácticas de las Matemáticas}

La experiencia de formar profesores en grado de Máster en Didáctica de las Matemáticas, se traduce en la "formación de hombres y mujeres con conocimientos de alto nivel matemático para resolver problemas de diversas índoles en la práctica educativa, utilizando el lenguaje y las herramientas que proporciona la didáctica de las matemáticas para el desarrollo de actividades docentes altamente calificado y la incorporación a equipos multidisciplinarios de investigación, especialmente, en la resolución de problemas, capacidad de análisis, síntesis y pensamiento abstracto" (URACCAN, 2016, p. 8). Los conocimientos, habilidades, actitudes y valores, que se asumen en el proceso de formación del máster en didácticas de las matemáticas, se caracterizan por las competencias siguientes:

- Marco normativo institucional, así como teoría y perspectiva de la investigación en didáctica de las matemáticas: políticas y normativas institucionales; didáctica de la educación matemática; e investigación en educación matemática.

- Investigación y desarrollo profesional; geometría y topología; didáctica de las matemáticas: e investigación en matemáticas y sus didácticas.

- Innovaciones tecnológicas para la enseñanza de las matemáticas: matemática computacional; matemáticas financieras; y estadística investigativa y operativa. 
- Evaluación, diseño curricular y desarrollo de tesis: evaluación educativa, diseño curricular; seminarios o coloquios de investigación.

Estas competencias contribuyen al profesorado a sistematizar su práctica docente, empleando de manera creativa los procesos de planeación, organización, ejecución y evaluación en la enseñanza y aprendizaje de las matemáticas, sustentados en los avances de la disciplina y sus didácticas; desarrollar capacidades y habilidades en la investigación e innovación en contextos multiculturales con perspectiva intercultural de género; acompañar y asesorar el trabajo en equipos interdisciplinarios para la articulación de diseños curriculares en y para la diversidad; y desarrollar conocimientos para la incorporación de las tecnologías en la enseñanza y aprendizaje de las matemáticas en contextos multiculturales (URACCAN, 2016).

\section{Tecnologías en la formación del profesorado de matemáticas para contextos multiculturales}

La nueva tendencia entre la matemática como área y la didáctica de las matemáticas como una didáctica específica y la evolución del campo tecnológico como herramienta que apoya los procesos de enseñanza y aprendizaje en el aula, favorece el desarrollo del aprendizaje de las matemáticas en contexto de diversidad. En la actualidad, las tecnologías han dado un nuevo enfoque a la forma de desarrollar la interacción del conocimiento en el aula, abriendo paso a la utilización de nuevas herramientas de apoyo a la docencia (León, 2014). Las tecnologías generan que el énfasis de la profesión docente se transforme desde una perspectiva centrada en el profesor y basada en clases magistrales hacia una formación centrada principalmente en el estudiantado dentro de unos ambientes de aprendizajes interactivos (UNESCO, 2004).

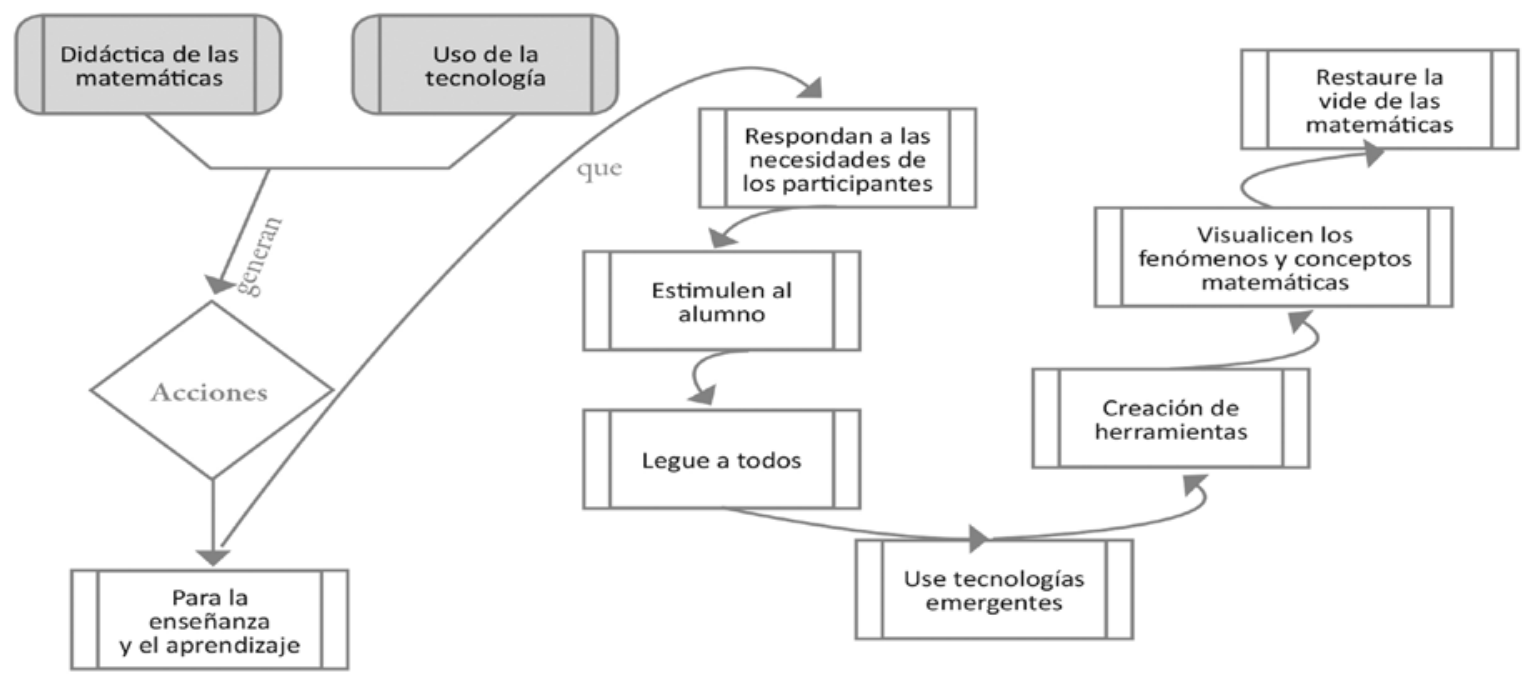

Figura 4: Relación entre didáctica y uso de las TIC (León, 2014).

Las tecnologías de la información y comunicación se han transformado en un elemento de innovación dentro del conocimiento humano. Es por ello, que el profesorado de matemáticas que integra las TIC, en la formación, es un docente: que lo hace como un modo de mejorar las experiencias de aprendizajes de los estudiantes; que conoce y maneja bien las tecnologías disponibles para apoyar su función; que utiliza las TIC para mejorar la gestión curricular en su ámbito de acción; que sirve las TIC como medio de inclusión social, de atención a la diversidad, realizando una actuación ética y legal respeto de su uso y cuidando la salud y del medio ambiente; que reconoce su responsabilidad para el estudiantado tenga un aprendizaje cada vez más eficiente y actual, incorporando las TIC; y que como profesores asuman responsablemente su propia actualización y desarrollo profesional con las potencialidades que presentan las TIC para su quehacer profesional (Flores \& Auzmendi, 2015b). 
Desde esta perspectiva, el profesorado de matemáticas incorpora tecnologías de la información y comunicación para la gestión del aprendizaje como un espacio de interacción e intercambio de experiencia que permite el uso de herramientas tecnológicas para gestionar, diseñar y evaluar actividades para el desarrollo del aprendizaje colaborativo, socio-afectivo y basado en problemas de la vida, que se concreta en ambientes e-learning; blended-learning; rapid e-learning; live e-learning; podcasting y Mobile-learning; y mundos virtuales que permiten la simulación del mundo real con sus mismas reglas, gravedad, su topografía, locomoción, acciones y comunicación.

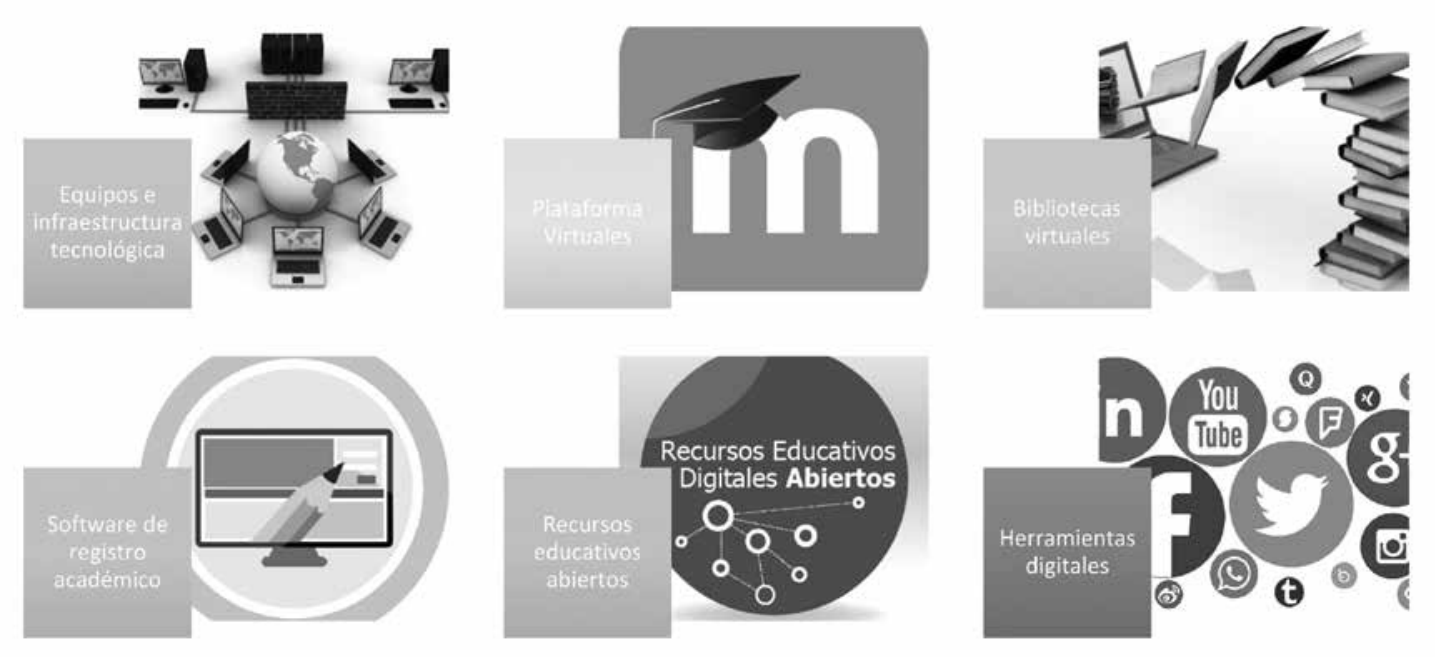

Figura 5: Las TIC en la formación del Profesorado de Matemáticas en la Universidad Comunitaria Intercultural.

Las tecnologías que se destacan para la enseñanza y aprendizaje de los conocimientos, saberes y prácticas matemáticas son: la implementación de equipos e infraestructura tecnológica; plataformas virtual Moodle; bibliotecas virtuales; software para el registro académico; recursos educativos abiertos; y herramientas digitales. Entre las herramientas digitales más destacadas son redes sociales (Facebook; G+; Twitter; LinkedIn; WhatsApp); Google Site; GoAnimate; Prezi; Slideshare; Educaplay; YouTube; y PowToon. Cabe mencionar que las TIC han fortalecido las capacidades de interacción y comunicación entre el profesorado y el estudiantado tomando en cuenta que la Educación Superior es un bien social y que debe estar acorde con los cambios que la sociedad vive experimentando (Flores \& Rojas, 2013).

También, se puede visualizar en los estudios de Flores (2017) sobre las actitudes del estudiantado universitario ante la incorporación de tecnologías en la enseñanza de las matemáticas, donde se encontró que las experiencias matemáticas con tecnologías se asociación con un coeficiente de correlación de Pearson de o,67, entendiéndose como el gusto para la integración de las TIC en la enseñanza y aprendizaje de las matemáticas. 
Esto se debe al modelo de formación de profesores de matemáticas en la Universidad Comunitaria Intercultural que se sustenta en ambientes interculturales y pluritecnológicos donde se incorporan entornos informáticos como GeoGebra, Wiris, R, SPSS y dispositivos tecnológicos como celulares con aplicaciones abiertas para calcular y graficar, así también calculadoras gráficas (Flores \& López-Mairena, 2016)

\section{Enseñanza y aprendizaje de las matemáticas en contextos multiculturales}

La enseñanza de las matemáticas en contextos multiculturales está orientada desde la pedagogía intercultural, entendiéndose como el desarrollo de un diálogo intercultural como una estrategia para promover espacio de innovación de nuevos conocimientos, ya que confronta elementos de diferentes horizontes y perspectivas culturales, abriendo la posibilidad de impulsar un proceso de complementación y enriquecimiento entre la ciencia moderna y otros saberes. Así, la formación del estudiantado en contextos multiculturales se configura como un proceso de negociabilidad de lo matemático en una comunidad de aprendizaje (León et al., 2013) en la que los aspectos siguientes se manifiestan y se tematizan: interculturalidad, perspectiva intercultural de género; autonomía regional; buen vivir y desarrollo con identidad; y diálogo de saberes y haceres (Flores, 2015).

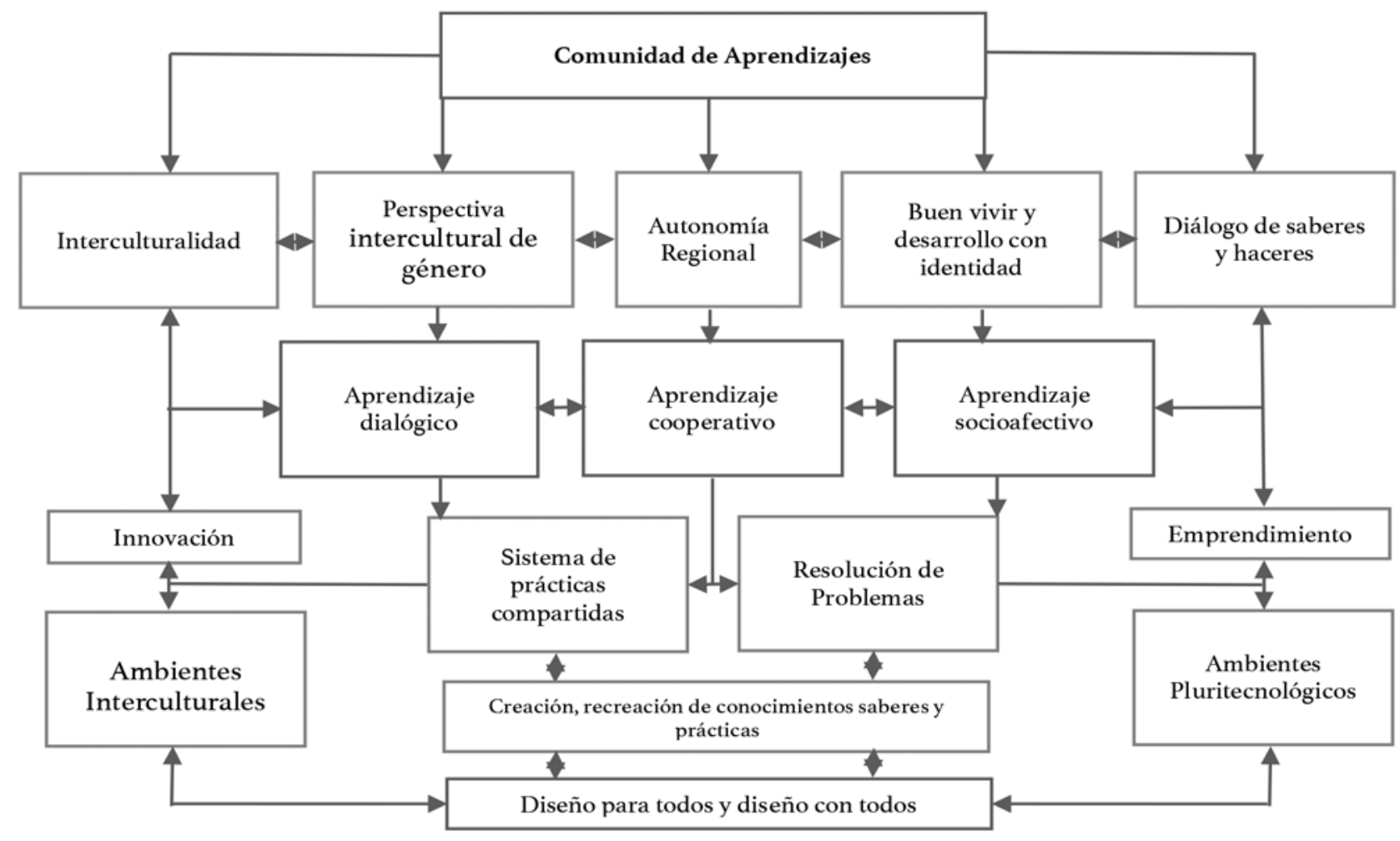

Figura 7: Modelo de enseñanzas de las matemáticas en contextos Multiculturales (Flores, 2016).

En esta dirección, la metodología se articula a partir de los fundamentos teóricos que ofrecen las pedagogías iluminadas por el enfoque histórico cultural. Por tal razón, se considera valioso orientar la formación hacia aspectos duales tales como: participación-cosificación, local y global, negociabilidad de significados-identificación, emergencia-diseño (Gil et al., 2013). En este sentido, es necesario que el profesorado produzca sus recursos didácticos y tecnológicos (Diseño para todos y diseño con todos) para que el estudiantado en cualquier condición sensorial, lingüística, cultural o socioeconómica interactuando juntos aprendan matemáticas y así el estudiantado se forma en ambiente de coexistencia con la diversidad de poblaciones (León et al., 2013). También, hay que tomar en cuenta que, en los recursos didácticos y tecnológicos se incorpora un sistema de prácticas compartidas, que se definen como el conjunto de accio- 
nes asociadas al hacer matemáticas, es decir, formular, probar, construir modelos, lenguajes, conceptos, teorías, intercambiar construcciones con otros y reconocer construcciones útiles a prácticas matemáticas en cada cultura (León, 2014).

Para enseñar matemáticas en contextos multiculturales es necesario tomar en cuenta la resolución de problemas a partir de las prácticas matemáticas y didácticas, entendiéndose práctica matemática y didáctica como cualquier acción o manifestación (lingüística o de otra forma) llevada a cabo por alguien para resolver problemas relacionados con las tareas profesionales y articuladoras de la práctica de enseñar matemáticas (León, 2014).

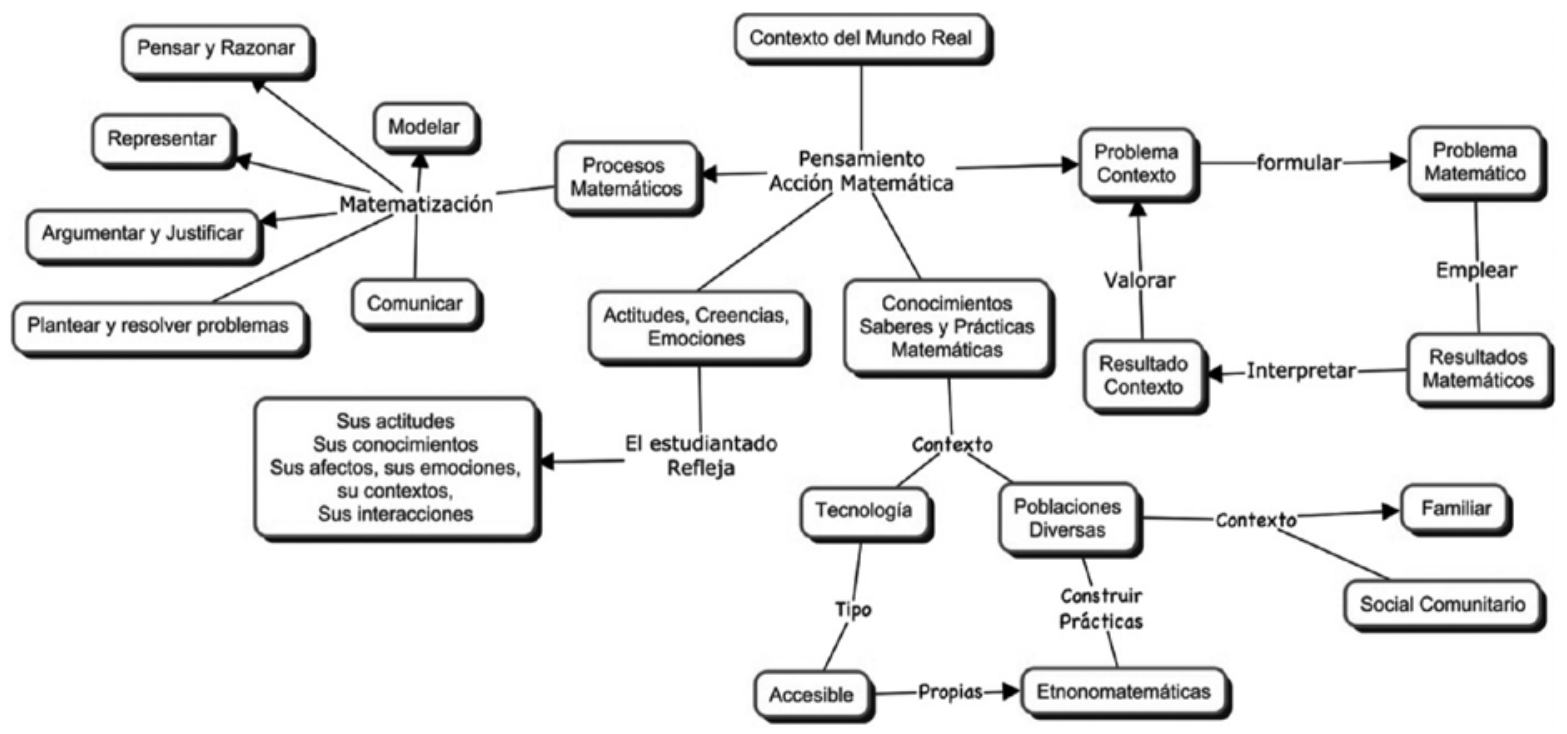

Figura 8: Modelo de resolución de problemas en contextos multiculturales (Flores, 2016).

También, se tiene que tomar en cuenta el proceso de matematización, este consiste en traducir los problemas desde el mundo real al matemático, por lo que se sustenta en varios subprocesos entre ellos: pensar y razonar; argumentar, justificar; comunicar; representar; modelar; representar; plantear y resolver problemas (NCTM, 2000; TIMSS, 2003; OECD, 2005). Los procesos matemáticos formalizan la resolución de problemas matemáticos. Según Pajares, Sanz y Rico (2004) su utilidad se concreta en establecer capacidades y habilidades específicas que ayudan a modular los objetivos, a establecer tareas escolares y caracterizar las propuestas de trabajo y las evaluaciones. Los contextos personal-familiar; social-comunitario; profesional-especializado; científico-tecnológico son aproximaciones fenomenológicas que constituyen ejes centrales que dotan de sentido al conocimiento matemático (Rico, 2006). Es decir, la variable contexto permite establecer la localización de un problema en términos de fenómenos de los que surgen la situación problemática considerada (OECD, 2004).

No obstante, en la enseñanza de las matemáticas en contextos multiculturales es necesario considerar las actitudes porque son instancias que predisponen y dirigen al sujeto sobre la realidad, filtran las percepciones y orientan el pensamiento para adaptarlo al contexto (Gairín, 1990). Las actitudes pueden expresarse mediante factores tales como: ideas, percepciones, gustos, preferencias, opiniones, creencias, emociones, sentimientos, comportamientos y tendencias a actuar. Tales factores han sido estudiados por diversos autores desde hace décadas, Gairín (1990), Auzmendi (1992), Gallego-Badillo (2000), GómezChacón (2000), Martínez (2008), Caballero, Guerrero y Blanco, (2014), Flores y Auzmendi (2015a), y Flores y Olivar (2016), y fueron organizados en función de componentes o dimensiones actitudinales: cognitivo (el conocer/ el saber); afectivo (la emoción/ el sentir); conativo o intencional (la intención); y comportamental (el comportamiento). 


\section{Referentes curriculares con incorporación tecnológica para la formación del profesora- do de matemáticas}

- El diseño, gestión y evaluación de referentes curriculares con incorporación tecnológica para la formación del profesorado de matemáticas, es un trabajo en comunidad de práctica que genera herramientas para la accesibilidad al estudiantado y la actualización didáctica-pedagógica y tecnológica al profesorado, así como, condiciones de una educación para todos y con todos a lo largo de toda la vida. Teniendo en cuenta que los referentes curriculares son el resultado del reconocimiento de cinco aspectos fundamentales: las múltiples experiencias con lo matemático y su didáctica; las múltiples representaciones de lo matemático y de su didáctica; los múltiples tipos de interacciones en los ambientes de aprendizaje; las poblaciones en vulnerabilidad educativa por su condiciones sensoriales, étnicas y económicas; y una metodología de interacción y desarrollo definida por las comunidades de prácticas. Según León (2016) los referentes curriculares se caracterizan por:

- Identificación de las problemáticas transversales en el contexto de la formación de profesores de matemáticas para atender poblaciones diversas.

- Se describe el papel y el funcionamiento de la comunidad de práctica como metodología de trabajo, en la construcción de los referentes curriculares.

- El marco de análisis de las relaciones pedagógicas y didácticas en educación matemática. Involucran las tecnologías empleadas en el contexto de la enseñanza y el aprendizaje de las matemáticas, las formas en que se manifiestan el compromiso de formar profesores de matemáticas en y para la diversidad.

- La reflexión y el consenso sobre la didáctica de las matemáticas, explicaciones teóricas, atendiendo a las condiciones pedagógicas y didácticas, sobre la cantidad, la magnitud y la forma, como campo de formación matemática necesaria presencia en los currículos de matemáticas y a sus relaciones con las diversas poblaciones involucradas en ALTER-NATIVA y con las TIC como diferentes a éstas.

- Los referentes se identifican y construyen: variantes e invariantes curriculares: ejes transversales en la formación de profesores, campos estructurantes específicos para la formación de profesores en matemáticas; y un conjunto de veintiún (21) referentes curriculares que articulan aspectos profesionales, pedagógicos y didácticas en la formación de profesores de matemáticas.

- Orientaciones para la operacionalización y utilización de los referentes. Se propone orientaciones para tres tipos de instrucciones y destinatarios del sistema educativo: los estamentos que crean y regulan las políticas educativas, las instituciones de Educación Superior, particularmente las facultades y programas de grado que forman profesores en las tres áreas mencionadas y la escuela específicamente los profesores en formación y en ejercicio.

Los referentes curriculares han constituido un proceso de construcción colectiva, es decir, un proceso de participación democrática e inclusiva, donde convergen diferentes instancias y representaciones con el fin de planificar, ejecutar y evaluar concertadamente, acciones de formación del profesorado de América Latina y el Caribe. 


\section{Conclusiones}

Este artículo ha descrito las experiencias innovadoras en la formación del profesorado en matemáticas en contextos multiculturales. La literatura nos indica las brechas educativas en la Costa Caribe de Nicaragua, así como indicadores de deserción universitaria y brechas de género entendida como sesgos culturales y educativos que fomentan el aprendizaje de los hombres y mujeres en relación con especialización de ciencias, tecnologías, ingeniería y matemáticas. Es por ello, que se analiza las innovaciones de formación del profesorado de matemáticas desde la perspectiva de la: formación en contextos multiculturales; formación en máster didáctica de las matemáticas; incorporación de tecnología; enseñar y aprender matemáticas; y referentes curriculares.

La formación del profesorado en contextos multiculturales es una estrategia de autodesarrollo regional para contrarrestar las condiciones de pobreza y marginación de los pueblos indígenas, afrodescendientes y mestizo de Nicaragua (Flores, 2015). Entre las experiencias innovadoras acumuladas en formación de profesores, están los grados en: Matemáticas y Física-Matemáticas. Estos profesores son formados desde los conocimientos pedagógico-didáctico, el reconocimiento de la diversidad y las características de un profesor en contextos multiculturales: creativo y emprendedor; investigador e innovador; facilitador y colaborativo; gestor de conocimientos, saberes y prácticas en comunidad.

La formación del profesorado en el grado de máster en didáctica de las matemáticas tiene como propósito promover la formación especializada y avanzada en Matemática que capacite a los futuros profesionales para la incorporación a grupos de investigación competitivos o para desarrollar procesos educativos de las matemáticas y sus didácticas. Cabe mencionar que esta innovadora formación contribuirá a los procesos de revitalización del conocimiento matemático de las mujeres, de los pueblos indígenas, afrodescendientes y mestizos, es decir, la maestría, ayudará a fomentar el rescate del conocimiento endógeno de pueblos originarios y promoverá la formación con perspectiva intercultural de género.

La incorporación de tecnologías en la formación del profesorado de matemáticas se ha caracterizado por ofrecer oportunidades de acceso para que el estudiantado interactúe e intercambie experiencias en un ambiente de aprendizaje innovador y colaborativo. Además, que integrar las tecnologías en la formación del profesores, a saber: que lo hace como un modo de mejorar las experiencias de aprendizaje del estudiantado (dimensión pedagógica); que conoce y maneja bien las tecnologías disponibles para apoyar su función docente (dimensión técnica e instrumental); que utiliza las tecnologías para mejorar la gestión curricular en su ámbito de acción (dimensión de gestión); que se sirve de las tecnologías como un medio de inclusión social, de atención a la diversidad, realizando una actuación ética y legal respecto de su uso y cuidando la salud y del medio ambiente (dimensión social, ética y legal); y finalmente, que reconoce su responsabilidad para que el estudiantado tenga un aprendizaje cada vez más eficiente y actual, usando o incorporando las tecnologías y como docente asuma responsablemente su propia actualización y desarrollo profesional con las potencialidades que presentan las tecnologías para su quehacer profesional (dimensión responsabilidad y desarrollo profesional).

Enseñar y aprender matemática en contextos multiculturales es a partir del enfoque intercultural, la metodología de comunidad de aprendizaje y la resolución de problemas, teniendo en cuenta que, en el caso de las mujeres, las poblaciones indígenas y afrodescendientes, es necesario identificar, describir y analizar las diferentes formas de hacer matemática de este género y estas poblaciones y de sus relaciones con las comunidades mestizas. También resulta ineludible explorar las formas o modos de etno-matemática que realizan para resolver sus problemas de la vida cotidiana, a fin de crear una propuesta acorde a la realidad de los discentes y docentes de estas comunidades. Aunque, el Estado de Nicaragua reconoce las diferencias lingüísticas, pero no asume una enseñanza a nivel de educación secundaria en lenguas indígenas, además que no invierte en la revitalización de estas lenguas y a la creación de palabras técnico-científico, para que estos estudiantes puedan asimilar los conceptos matemáticos avanzados. 
En definitiva, el lenguaje juega un papel importante en la comprensión de los conocimientos matemáticos, por eso es necesario que en el uso de la lengua natural y de otros sistemas de representación se propicien un desarrollo de las condiciones intelectuales y sociales de los sujetos. Es decir, hay que integrar el capital lingüístico, además del cultural, social y económico de los estudiantes en la construcción del quehacer de las instituciones académicas y particularmente en el proceso de enseñanza y aprendizaje de las matemáticas.

\section{Lista de referencia}

ACACIA. (2015). Centros de Cooperación para el Fomento, Fortalecimiento y Transferencia de Buenas Prácticas que Apoyan, Cultivan, Adaptan, Comunican, Innovan y Acogen a la Comunidad Universitaria. Bogotá: Universidad Distrital Franscico José de Caldas.

Auzmendi, E. (1992). Las actitudes hacia la matemática-estadística en la enseñanzas medias y universitarias. Bilbao: Mensajero.

Ball, D., Thames, M., \& Phelps, G. (2008). Content knowledge for teaching: What makes it special? Journal of Teacher Education, 59(5), 389-407.

Bisquerra, R. (2009). Metodología de la Investigación Educativa. Madrid: La Muralla.

Caballero, A., Guerrero, E., \& Blanco, L. (2014). Construcción y administración de un instrumento para la evaluación de los afectos hacia las matemáticas. Campo Abierto, 33(1), 47-71.

CEPAL. (2016). Agenda 2030 y los objetivos de desarrollo sostenibles: Una oportunidad para América Latina y el Caribe. Santiago: Naciones Unidas.

CNU. (2016). Informe de rendición de cuentas 2015. Managua: CNU.

EDUQUEMOS (2011). Situación y perspectiva en Nicaragua para Universalizar Primaria de Calidad. Eduquemos. Managua: Eduquemos Pedagógicos.

EDUQUEMOS(2014). Calidad y Equidad para el Desarrollo Humano: Informe de progreso educativo Nicaragua. Managua: Fundación Telefónica.

Flores, W., O. (2015). La formación del profesorado de matemática desde el ámbito de la Universidad Comunitaria Interculturla. Ciencia e Interculturalidad, 16(1), 32-53. DOI: http://dx.doi.org/10.5377/ rci.v16i1.2352

Flores, W., O. (2016). Análisis ontosemiótico en los procesos de resolución de problemas por estudiantes universitarios. Tesis Doctoral. Bilbao: Universidad de Deusto.

Flores, W., O. (2017). Actitudes del estudiantado universitario ante la incorporación de tecnologías en la enseñanza de las matemáticas. Horizontes Pedagógicos, 19(1), 8-18.

Flores, W., O., \& Auzmendi, E. (2015a). Análisis de la estructura factorial de una escala de actitud hacia las matemáticas. Aula de Encuentro, 17(1), 45-77.

Flores, W., O., \& Auzmendi, E. (2015b). Integración de las Tecnologías de la Información y Comunicación en la Formación de Profesores de Matemática en y para la diversidad. En C. Varela, A. Miñán, \& L. Bengochea (Ed.), Congreso Internacional Sobre Formación virtual inclusiva y de calidad para el siglo XXI (281288). Granada: Universidad de Granada. ISBN 978-84-338-5744-6. 
Flores, W., O., \& López, E. (2016). Recursos didácticos y tecnológicos para la enseñanza de la integral definida en el modelo de Universidad Comunitaria Intercultural . Ciencia e Interculturalidad, 18(1), 63-78. DOI: http://dx.doi.org/10.5377/rci.v18i1.3050

Flores, W., O., Gutiérrez y Restrepo, E., León, O., Sarraipa, J., Lima, C., Merino, C., Calderón, I., Guinocchio, M., Rivera, M., Calderón, M., \& Boticario, J. (2016). Centros de apoyo y desarrollo educativo profesional para la disminución de la deserción universitaria. Ciencia e Interculturalidad, 18(1), 48-62.

Flores, W., O., \& Olivar, S., A. (2016). Actitudes hacia la estadística en la formación del profesorado para contextos multiculturales. Revista Universitaria del Caribe, 17(2), 36-45. DOI: http://dx.doi. org/10.5377/ruc.v17i2.3235

Flores, W., O., \& Rojas, N. (2013). Integración de las Tecnologías de la Información y Comunicación en la Enseñanza Universitaria. Horizontes Pedagógicos, 15(1), 46-57.

Flórez, C. A. (2012). La Educación Primaria: Condiciones que favorecen u obstaculizan el aumento de la matricula, la retención y la promoción escolar. Managua: IEEPP.

Gairín, J. (1990). Las actitudes en educación. Un estudio sobre la educación matemática. Barcelona: Boixareu.

Gallego-Badillo, R. (200o). Los problemas de las competencias cognoscitivas. Una discusión necesaria. Bogotá: Universidad Pedagógica Nacional.

Gil, D., Sáiz, M., Cavanzo, A., León, O., Medina, R., Bonilla, M, Romero, J., Correal, M., Flores, W., O., Rojas, N., Peralta, M., Baca, J., Avila, C., \& Márquez, A. (2013). Una experiencia de construcción de recursos educativos abiertos, para la formación de profesores de matemáticas en contexto de diversidad en el proyecto ALTER-NATIVA. En A. Moreira, L. Bengochea, \& J. Hilera (Ed.), Congreso Internacional para una formación virtual accesible y de calidad (170-177). Lisboa: Universidad de Lisboa. ISBN: 978-989-8553-18-8

Gil, N., Blanco, L., \& Guerrero, E. (2005). El dominio afectivo en el aprendizaje de las matemáticas. Una revisión de sus descriptores básicos. UNIÓN. Revista Iberoamericana de Educación Matmeática, 2, 15-32.

Gómez-Chacón, I. (2000). Matemática emocional. Los afectos en el aprendizaje matemático. Madrid: Narcea.

Hernández, R., Fernández, C., \& Baptista, M. (2010). Metodología de la Investigación. México: McGra-Hill.

León, O. (2014). Referentes curriculares con incorporación tecnológica para la formación del profesorado de matemáticas en y para la diversidad. Bogotá: Universidad Distrital Francisco José de Caldas.

León, O. (2016). Referentes curriculares con incorporación tecnológica para la formación de profesores de matemática en y para la diversidad. Ciencia e Interculturalidad , 18(1), 34-47. DOI: http://dx.doi. org/10.5377/rci.v18i1.3048

León, O., Medina, R., Saiz, M., Bonilla, M., Romero, J., Gil, D., Correal, M., Flores, W., O., Rojas, N., Peralta, M., Cavanzo, A., Baca, J., Avila, C., \& Márquez, A. (2013). Relaciones entre "Diseño para Todos" $y$ "Diseño con Todos" en Formación de Profesores de Matemáticas. En A. Moreira, L. Bengochea, \& J. Hilera (Ed.), Congreso Internacional Para una Formación Virtual Accesible y de Calidad (162-169). Portugal: Universidad de Lisboa. ISBN: 978-989-8553-18-8

Martín, E., Dolz, D., \& Mauri, T. (1997). La atención a la diversidad en la educación secundaria. Barcelona: Ice Horsori. 
Martínez, O. (2008). Actitud hacia la matemática. Sapiens: Revista Universitaria de Investigación, 237-256.

NCTM. (2000). Principles and standards for school mathematics. VA: NCTM.

OECD. (2004). Learning for Tomorrow's World: First results from PISA 2003. Paris: OECD.

OECD. (2005). Informe PISA 2003. Aprender para el mundo de mañana. Madrid: Santillana.

Pajarez, R., Sanz, A., \& Rico, L. (2004). Aproximación a un modelo de evaluación: el proyecto PISA 2000. Madrid: Ministerio de Educación, Cultura y Deporte.

Rico, L. (2006). Marco teórico de evaluación PISA sobre matemáticas y resolución de problemas. Revista de Educación Matemática, Extraordinario, 275-294.

Sandin, M. (2003). Investigación cualitativa en investigación. Fundamentos y Tradiciones. Madrid: McGraw-Hill.

Shulman, S. (1986). Those who understand: Knowledge growth in teaching. Educational Research, 15, 4-14.

TIMSS (2003). Marcos teóricos y especificamente de evaluación TIMSS 2003. Madrid: Ministerio de Educación, Cultura y Deporte-INCE.

UNESCO. (2004). Las tecnologías de la información y comunicación en la formación docente. Montevideo: Ediciones Trilce.

URACCAN (2010). Estudios de seguimiento a graduados y graduadas de URACCAN, generación 2001-2007 del nivel de licenciatura e ingeniería. Costa Caribe de Nicaragua: URACCAN.

URACCAN (2016). Programa Curricular de la Maestría en Didácticas de las Matemáticas. Nueva Guinea, Nicaragua: URACCAN.

USAID (2012). Nicaragua Gender Analysis. Managua: USAID.

USAID (2013). Nicaragua Country Development Cooperation Strategy. Managua: USAID. 